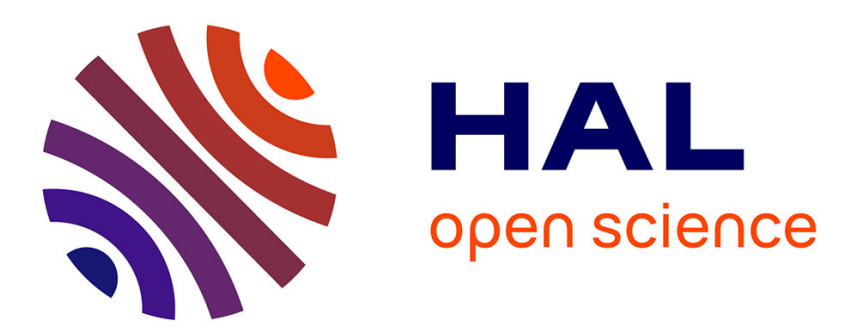

\title{
Non-linear dynamics of a whole vehicle FE Model using a Harmonic Balance Method
}

Franck Barillon, Jean-Jacques Sinou, Jean-Marc Duffal, Louis Jézéquel

\section{To cite this version:}

Franck Barillon, Jean-Jacques Sinou, Jean-Marc Duffal, Louis Jézéquel. Non-linear dynamics of a whole vehicle FE Model using a Harmonic Balance Method. International Journal of Vehicle Design, 2013, 63 (4), pp.387-403. 10.1504/IJVD.2013.057471 . hal-00914409

\section{HAL Id: hal-00914409 \\ https://hal.science/hal-00914409}

Submitted on 7 Jun 2014

HAL is a multi-disciplinary open access archive for the deposit and dissemination of scientific research documents, whether they are published or not. The documents may come from teaching and research institutions in France or abroad, or from public or private research centers.
L'archive ouverte pluridisciplinaire HAL, est destinée au dépôt et à la diffusion de documents scientifiques de niveau recherche, publiés ou non, émanant des établissements d'enseignement et de recherche français ou étrangers, des laboratoires publics ou privés. 
Non-linear dynamics of a whole vehicle FE Model using a Harmonic Balance Method

\title{
F. Barillon
}

Laboratoire de Tribologie et de Dynamique des Systèmes

UMR CNRS 5513, Ecole Centrale de Lyon,

36, avenue Guy de Collongue,

69134 Ecully Cedex, France

Renault SA, Acoustic Research Department

1, avenue du Golf

78288 GUYANCOURT

E-mail: franck.barillon@renault.com

*Corresponding author

\section{J.J. Sinou*}

Laboratoire de Tribologie et de Dynamique des Systèmes UMR CNRS 5513, Ecole Centrale de Lyon,

36, avenue Guy de Collongue,

69134 Ecully Cedex, France

E-mail: jean-jacques.sinou@ec-lyon.fr

\section{J.M. Duffal}

Renault SA, Acoustic Research Department

1, avenue du Golf

78288 GUYANCOURT

E-mail: jean-marc.duffal@renault.com

\section{Jézéquel}

Laboratoire de Tribologie et de Dynamique des Systèmes

UMR CNRS 5513, Ecole Centrale de Lyon,

36, avenue Guy de Collongue,

69134 Ecully Cedex, France

E-mail: louis.jezequel@ec-lyon.fr

\begin{abstract}
The aim of the present paper is to apply the Harmonic Balance Method (HBM) to a Finite Element Model of a complete vehicle (body, engine and engine mounts) in order to calculate the non-linear response of the assembly. The non-linear effects come from the amplitude-dependent stiffness of the engine mounts. First, the Harmonic Balance Method is presented. A condensation process on the non-linear degrees-of-freedom is also proposed. This process
\end{abstract}


reduces the original non-linear system by focusing only on the solution of the non-linear equations associated with the system's non-linear components. Secondly, the engine mount stiffness dependency with amplitude is measured on a test bench to estimate a polynomial stiffness law. Finally, the numerical analysis is performed to analyze the non-linear response of the whole vehicle using the Harmonic Balance Method algorithm with appropriate condensation located only on the non-linear coordinates of the system in order to minimize computer time.

Keywords: Harmonic Balance Method; Engine mounts; Vehicle Finite Element Model; Amplitude-dependent stiffness; Craig and Bampton condensation procedure; condensation on non-linear DOFs.

\section{Introduction}

A whole vehicle is a complex assembly of many subsystems : the car body, the engine, the front and rear axles, etc. Both experimental and numerical expertise has been developed to predict the response of each isolated subsystem that remains linear on usual frequency ranges of study. All these subsystems are coupled by elastic components which have two main functions: to maintain subsystems together and to limit the transmission of vibrations.

These elastic components are often represented as simple linear stiffness and damping, linking subsystems together. However, they are made of metal, viscoelastic fluids and rubber and thus have very complex mechanical behaviour. They may be strongly non-linear with different environment parameters such as frequency, temperature, static preload or amplitude.

In this paper we study the coupling between the engine and the car body. In most of cars, the link between the engine and the car body is based on a pendular engine mounting system presented Figure 1. This system includes an engine mount, a gearbox mount, a lower torque rod and an upper torque rod for powerful engines.

In Section 2 of this paper we present the Harmonic Balance Method with a condensation process on the non-linear degrees-of-freedom that allows computing the non linear dynamics of a system containing non-linear components (J.J. Sinou (2009);E.J. Hahn and P.Y. Chen (1989);V. Jaumouillé, J.J. Sinou and B. Petitjean (2010);J. Tezcan and J-K Hsiao (2008)).

In Section 3, we apply the Harmonic Balance Method to a whole vehicle FE model to compute the non-linear dynamic of the car excited by a simple harmonic force. The non-linear behaviour of the engine mounts with amplitude is measured on a test bench and we estimate a polynomial law for the amplitude-dependent stiffness. 
Figure 1 Pendular engine mounting system

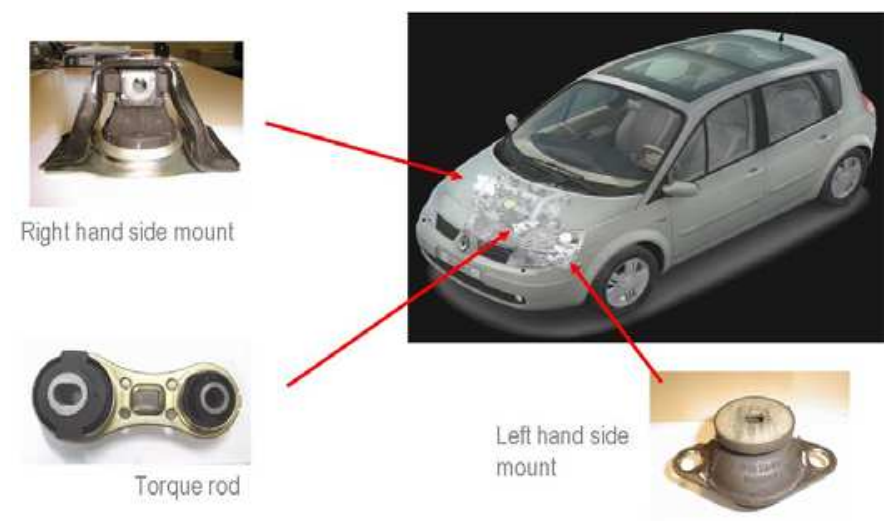

\section{General theory of the Harmonic Balance Method and condensation process}

In this section, the Harmonic Balance Method with a condensation process on the non-linear degrees-of-freedom will be presented.

\subsection{The Harmonic Balance Method}

For a mechanical system, the equations of motion may be written as

$$
\begin{aligned}
\boldsymbol{M} \ddot{\boldsymbol{X}}(t)+\boldsymbol{D} \dot{\boldsymbol{X}}(t)+\boldsymbol{K} \boldsymbol{X}(t) & =\boldsymbol{F}(t)+\boldsymbol{F}_{L}(\boldsymbol{X}(t), \dot{\boldsymbol{X}}(t), \omega, t)+\boldsymbol{F}_{N L}(\boldsymbol{X}(t), \dot{\boldsymbol{X}}(t), \omega, t) \\
& =\boldsymbol{F}(\boldsymbol{X}(t), \dot{\boldsymbol{X}}(t), \omega, t)
\end{aligned}
$$

where $\boldsymbol{F}_{L}$ and $\boldsymbol{F}_{N L}$ are the linear and non-linear forces located on the linear and the non-linear degrees-of-freedom, respectively. $\boldsymbol{F}(t)$ represents the external or internal periodic force of the system. $\boldsymbol{X}(t)$ represents the time-dependent solution of size $n . \boldsymbol{M}, \boldsymbol{K}$ and $\boldsymbol{D}$ represent the mass matrix, the stiffness matrix and the damping matrix, respectively. In order to estimate the response of the non-linear system as a truncated Fourier series (if this solution exists), the right-hand side of the system is assumed to be a function that is periodic in time with period $T$. Thus we assume that the non-linear dynamical response of the system may be approximated by finite Fourier series with $\Omega=\frac{2 \pi}{T}$ the fundamental frequency:

$$
\boldsymbol{X}(t)=\boldsymbol{B}_{0}+\sum_{k=1}^{m} \boldsymbol{A}_{k} \sin (k \Omega t)+\boldsymbol{B}_{k} \cos (k \Omega t)
$$

where $m$ is the order of the Fourier series and $\boldsymbol{B}_{0}, \boldsymbol{A}_{k}$ and $\boldsymbol{B}_{k}$ define the coefficients of the finite Fourier series. The number of harmonic coefficients is selected on the basis of the number of significant harmonics expected in 
the non-linear dynamical response. Moreover, we assume that the force vector $\boldsymbol{F}(\boldsymbol{X}(t), \dot{\boldsymbol{X}}(t), \omega, t)$ can be approximated by finite Fourier series of order $m$

$$
\boldsymbol{F}(t)=\boldsymbol{C}_{0}+\sum_{k=1}^{m} \boldsymbol{S}_{k} \sin (k \Omega t)+\boldsymbol{C}_{k} \cos (k \Omega t)
$$

Substituting equation 2 and equation 3 in equation 1 yields a set of $(2 m+1) * n$ equations. Using the first $n^{\text {th }}$ equations, the constant terms $\boldsymbol{B}_{0}$ can be determined by equation 4

$$
\boldsymbol{K} \boldsymbol{B}_{0}=\boldsymbol{C}_{0}
$$

For each order $k$, the $k^{t h}$ Fourier coefficients $\boldsymbol{A}_{k}$ and $\boldsymbol{B} k$ are given by equation 5

$$
\left[\begin{array}{cc}
\boldsymbol{K}-\Omega^{2} \boldsymbol{M} & -k \Omega \boldsymbol{D} \\
k \Omega \boldsymbol{D} & \boldsymbol{K}-\Omega^{2} \boldsymbol{M}
\end{array}\right]\left\{\begin{array}{l}
\boldsymbol{A}_{k} \\
\boldsymbol{B}_{k}
\end{array}\right\}=\left\{\begin{array}{l}
\boldsymbol{S}_{k} \\
\boldsymbol{C}_{k}
\end{array}\right\} \quad \forall k \in[1, m]
$$

However, in case of complex non-linearity the Fourier coefficients $\boldsymbol{C}_{0}, \boldsymbol{S}_{k}$ and $\boldsymbol{C}_{k}$ $(\forall k \in[1, m])$ are not directly functions of $\boldsymbol{B}_{0}, \boldsymbol{A}_{k}$ and $\boldsymbol{B}_{k}(\forall k \in[1, m])$. T.M. Cameron, J.H. Griffin (1989) suggested that the truncated Fourier expression of $\boldsymbol{F}(\boldsymbol{X}(t), \dot{\boldsymbol{X}}(t), \omega, t)$ should be calculated by applying an Alternate/Frequency Time domain method (AFT-method) :

$$
\left[\begin{array}{c}
\boldsymbol{B}_{0} \\
\boldsymbol{A}_{1} \\
\boldsymbol{B}_{1} \\
\vdots \\
\boldsymbol{A}_{m} \\
\boldsymbol{B}_{m}
\end{array}\right] \stackrel{F F T}{\rightarrow} \boldsymbol{X}(t) \rightarrow \boldsymbol{F}(\boldsymbol{X}(t), \dot{\boldsymbol{X}}(t), \omega, t) \stackrel{F F T^{-1}}{\rightarrow}\left[\begin{array}{c}
\boldsymbol{C}_{0} \\
\boldsymbol{S}_{1} \\
\boldsymbol{C}_{1} \\
\vdots \\
\boldsymbol{S}_{m} \\
\boldsymbol{C}_{m}
\end{array}\right]
$$

Following this, the $(2 m+1) * n$ non-linear equations of motion 4 and 5 can be solved by using a non-linear system of equations solver such as the Broyden method (C.G. Broyden (1994)).

\subsection{Condensation procedure}

If a non-linear system consists in $n$-degrees-of-freedom system with non-linear forces associated with $q$ of these elements, this system may be considered as a linear structure with $p=n-q$ degrees-of-freedom and having several additional non-linear elements. Therefore it may be of great interest to keep only the $q$ nonlinear degrees-of-freedom. Equation 1 can be re-ordered by considering the linear transformation $\boldsymbol{X}=\boldsymbol{P} \boldsymbol{Y}=\boldsymbol{P}\left[\boldsymbol{Y}_{q} \boldsymbol{Y}_{p}\right]$ where $\boldsymbol{Y}_{q}$ and $\boldsymbol{Y}_{p}$ contain the $q$ non-linear degrees-of-freedom and the $p$ linear degrees-of-freedom, respectively. Therefore, the non-linear equation 1 is transformed by

$$
\left[\begin{array}{cc}
\tilde{\boldsymbol{M}}_{q q} & \tilde{\boldsymbol{M}}_{q p} \\
\tilde{\boldsymbol{M}}_{p q} & \tilde{\boldsymbol{M}}_{p p}
\end{array}\right]\left\{\begin{array}{c}
\ddot{\boldsymbol{Y}}_{q}(t) \\
\ddot{\boldsymbol{Y}}_{p}(t)
\end{array}\right\}+\left[\begin{array}{cc}
\tilde{\boldsymbol{D}}_{q q} & \tilde{\boldsymbol{D}}_{q p} \\
\tilde{\boldsymbol{D}}_{p q} & \tilde{\boldsymbol{D}}_{p p}
\end{array}\right]\left\{\begin{array}{c}
\dot{\boldsymbol{Y}}_{q}(t) \\
\dot{\boldsymbol{Y}}_{p}(t)
\end{array}\right\}+\left[\begin{array}{cc}
\tilde{\boldsymbol{K}}_{q q} & \tilde{\boldsymbol{K}}_{q p} \\
\tilde{\boldsymbol{K}}_{p q} & \tilde{\boldsymbol{K}}_{p p}
\end{array}\right]\left\{\begin{array}{c}
\boldsymbol{Y}_{q}(t) \\
\boldsymbol{Y}_{p}(t)
\end{array}\right\}=\left\{\begin{array}{c}
\boldsymbol{F}_{q}(t) \\
\boldsymbol{F}_{p}(t)
\end{array}\right\}(7)
$$

Here, we present the condensation procedure used to obtain the Fourier coefficients associated with the non-linear and linear elements of the complete system. 
2.2.1 Determination of the constant terms associated with the non-linear elements

By substituting equation 2 and equation 3 in equation 7 , and by only equating coefficients for the constant terms corresponding to the first linear equation of the system 4 , we obtain

$$
\left[\begin{array}{ll}
\tilde{\boldsymbol{K}}_{q q} & \tilde{\boldsymbol{K}}_{q p} \\
\tilde{\boldsymbol{K}}_{p q} & \tilde{\boldsymbol{K}}_{p p}
\end{array}\right]\left\{\begin{array}{l}
\boldsymbol{B}_{0}^{q} \\
\boldsymbol{B}_{0}^{p}
\end{array}\right\}=\left\{\begin{array}{l}
\boldsymbol{C}_{0}^{q} \\
\boldsymbol{C}_{0}^{p}
\end{array}\right\}
$$

where $\boldsymbol{B}_{0}^{q}$ and $\boldsymbol{B}_{0}^{p}$ are the vectors with the $q$ non-linear and the $p$ linear degrees-offreedom of the system, respectively. By eliminating $\boldsymbol{B}_{0}^{p}$ from the latter equation, $\boldsymbol{B}_{0}^{q}$ is given by

$$
\boldsymbol{B}_{0}^{q}=\left(\tilde{\boldsymbol{K}}_{q q}-\tilde{\boldsymbol{K}}_{q p} \tilde{\boldsymbol{K}}_{p p}^{-1} \tilde{\boldsymbol{K}}_{p q}\right)^{-1}\left(\boldsymbol{C}_{0}^{q}-\tilde{\boldsymbol{K}}_{q p} \tilde{\boldsymbol{K}}_{p p}^{-1} \boldsymbol{C}_{0}^{p}\right)
$$

\subsubsection{Determination of the sine Fourier coefficients associated with the non-} linear elements

Equation 5 may be written as

$$
\begin{aligned}
\left(\tilde{\boldsymbol{K}}-(k \Omega)^{2} \tilde{\boldsymbol{M}}\right) \boldsymbol{A}_{k}-k \Omega \tilde{\boldsymbol{D}} \boldsymbol{B}_{k} & =\boldsymbol{S}_{k} \\
k \Omega \tilde{\boldsymbol{D}} \boldsymbol{A}_{k}+\left(\tilde{\boldsymbol{K}}-(k \Omega)^{2} \tilde{\boldsymbol{M}}\right) \boldsymbol{B}_{k} & =\boldsymbol{C}_{k}
\end{aligned}
$$

By eliminating coefficients $\boldsymbol{B}_{k}$ in equation 10 thanks to equation 11 we obtain

$$
\left[\tilde{\boldsymbol{K}}-(k \Omega)^{2} \tilde{\boldsymbol{M}}+k \Omega \tilde{\boldsymbol{D}}\left(\tilde{\boldsymbol{K}}-(k \Omega)^{2} \tilde{\boldsymbol{M}}\right)^{-1} k \Omega \tilde{\boldsymbol{D}}\right] \boldsymbol{A}_{k}=\boldsymbol{S}_{k}+k \Omega \tilde{\boldsymbol{D}}\left(\tilde{\boldsymbol{K}}-(k \Omega)^{2} \tilde{\boldsymbol{M}}\right)^{-1} \boldsymbol{C}_{k}(
$$

By introducing

$$
\begin{aligned}
& \boldsymbol{T}_{k}=\left[\tilde{\boldsymbol{K}}-(k \Omega)^{2} \tilde{\boldsymbol{M}}+k \Omega \tilde{\boldsymbol{D}}\left(\tilde{\boldsymbol{K}}-(k \Omega)^{2} \tilde{\boldsymbol{M}}\right)^{-1} k \Omega \tilde{\boldsymbol{D}}\right] \\
& \boldsymbol{W}_{k}=\boldsymbol{S}_{k}+k \Omega \tilde{\boldsymbol{D}}\left(\tilde{\boldsymbol{K}}-(k \Omega)^{2} \tilde{\boldsymbol{M}}\right)^{-1} \boldsymbol{C}_{k}
\end{aligned}
$$

And after separating non-linear and linear degrees-of-freedom, equation 12 may be re-written as

$$
\left[\begin{array}{ll}
\boldsymbol{T}_{k}^{q q} & \boldsymbol{T}_{k}^{q p} \\
\boldsymbol{T}_{k}^{p q} & \boldsymbol{T}_{k}^{p p}
\end{array}\right]\left\{\begin{array}{l}
\boldsymbol{A}_{k}^{q} \\
\boldsymbol{A}_{k}^{p}
\end{array}\right\}=\left\{\begin{array}{l}
\boldsymbol{W}_{k}^{q} \\
\boldsymbol{W}_{k}^{p}
\end{array}\right\}
$$

By eliminating coefficients $\boldsymbol{A}_{k}^{p}$, coefficients $\boldsymbol{A}_{k}^{q}$ are given by

$$
\boldsymbol{A}_{k}^{q}=\left(\boldsymbol{T}_{k}^{q q}-\boldsymbol{T}_{k}^{q p} \boldsymbol{T}_{k}^{p p-1} \boldsymbol{T}_{k}^{p q}\right)^{-1}\left(\boldsymbol{W}_{k}^{q}-\boldsymbol{T}_{k}^{q p} \boldsymbol{T}_{k}^{p p-1} \boldsymbol{W}_{k}^{p}\right)
$$


2.2.3 Determination of the cosine Fourier coefficients associated with the non-linear elements

Starting from equation 11 and eliminating coefficients $\boldsymbol{A}_{k}$ thanks to equation 10 we obtain

$$
\left[\tilde{\boldsymbol{K}}-(k \Omega)^{2} \tilde{\boldsymbol{M}}+k \Omega \tilde{\boldsymbol{D}}\left(\tilde{\boldsymbol{K}}-(k \Omega)^{2} \tilde{\boldsymbol{M}}\right)^{-1} k \Omega \tilde{\boldsymbol{D}}\right] \boldsymbol{B}_{k}=\boldsymbol{C}_{k}-k \Omega \tilde{\boldsymbol{D}}\left(\tilde{\boldsymbol{K}}-(k \Omega)^{2} \tilde{\boldsymbol{M}}\right)^{-1} \boldsymbol{S}_{k}(17)
$$

By introducing equation 13 and the following relationship

$$
\boldsymbol{U}_{k}=\boldsymbol{C}_{k}-k \Omega \tilde{\boldsymbol{D}}\left(\tilde{\boldsymbol{K}}-(k \Omega)^{2} \tilde{\boldsymbol{M}}\right)^{-1} \boldsymbol{S}_{k}
$$

We obtain

$$
\left[\begin{array}{ll}
\boldsymbol{T}_{k}^{q q} & \boldsymbol{T}_{k}^{q p} \\
\boldsymbol{T}_{k}^{p q} & \boldsymbol{T}_{k}^{p p}
\end{array}\right]\left\{\begin{array}{l}
\boldsymbol{B}_{k}^{q} \\
\boldsymbol{B}_{k}^{p}
\end{array}\right\}=\left\{\begin{array}{l}
\boldsymbol{U}_{k}^{q} \\
\boldsymbol{U}_{k}^{p}
\end{array}\right\}
$$

By eliminating coefficients $\boldsymbol{B}_{k}^{p}$, coefficients $\boldsymbol{B}_{k}^{q}$ are given by

$$
\boldsymbol{B}_{k}^{q}=\left(\boldsymbol{T}_{k}^{q q}-\boldsymbol{T}_{k}^{q p} \boldsymbol{T}_{k}^{p p-1} \boldsymbol{T}_{k}^{p q}\right)^{-1}\left(\boldsymbol{U}_{k}^{q}-\boldsymbol{T}_{k}^{q p} \boldsymbol{T}_{k}^{p p-1} \boldsymbol{U}_{k}^{p}\right)
$$

\subsubsection{Determination of the Fourier coefficients associated with the linear} elements

The linear $(2 m+1) * p$ Fourier coefficients $\boldsymbol{B}_{0}^{p}, \boldsymbol{A}_{k}^{p}$ and $\boldsymbol{B}_{k}^{p}$ can be estimated from equations 8, 15 and 19. After calculations, we obtain

$$
\begin{aligned}
\boldsymbol{B}_{0}^{p} & =\tilde{\boldsymbol{K}}_{p p}^{-1}\left(\boldsymbol{C}_{0}^{p}-\tilde{\boldsymbol{K}}_{p q} \boldsymbol{B}_{0}^{q}\right) \\
\boldsymbol{A}_{k}^{p} & =\boldsymbol{T}_{k}^{p p-1}\left(\boldsymbol{W}_{k}^{p}-\boldsymbol{T}_{k}^{p q} \boldsymbol{A}_{k}^{q}\right) \\
\boldsymbol{B}_{k}^{p} & =\boldsymbol{T}_{k}^{p p-1}\left(\boldsymbol{U}_{k}^{p}-\boldsymbol{T}_{k}^{p q} \boldsymbol{B}_{k}^{q}\right)
\end{aligned}
$$

\subsubsection{Complete process for the determination of the Fourier coefficients and the non-linear dynamical response of the system}

In conclusion, the Fourier coefficients $\boldsymbol{B}_{0}^{q}, \boldsymbol{A}_{k}^{q}$ and $\boldsymbol{B}_{k}^{q}$ associated with the nonlinear components of the system are first determined by considering the $(2 \mathrm{~m}+$ 1) $* q$ equations 9, 16 and 20. These equations are solved by a solver such as the Broyden method (C.G. Broyden (1994)). Secondly, determination of the $\boldsymbol{B}_{0}^{p}$, $\boldsymbol{A}_{k}^{p}$ and $\boldsymbol{B}_{k}^{p}$ associated with the linear components of the system are obtained by considering equations 21, 22 and 23. We can note that the iterative resolution is only performed on the non-linear degrees-of-freedom. The response on the linear degrees-of-freedom is then an explicit function of the response on the non-linear degrees-of-freedom.

Some linear degrees-of-freedom of the vector $\boldsymbol{Y}_{p}$ can be transferred and added to the vector $\boldsymbol{Y}_{q}$ of the non-linear degrees-of-freedom without loosing the general 
process presented previously. This operation can be very interesting if keeping the physical linear degrees-of-freedom is necessary for the study (i.e. the non-linear behavior of the chosen physical linear degrees-of-freedom can be obtained directly by using relations 16 and 20, without calculating expressions 22 and 23). However, in this case, the size of the vector $\boldsymbol{Y}_{q}$ has been increased, thereby increasing calculation time and storage requirements.

\section{Application to a whole vehicle FE Model}

In this section, the HBM method will be applied on a whole vehicle FE Model containing non-linear engine mounts stiffness with amplitude.

\subsection{Amplitude-dependent stiffness measurement for the engine mounts}

\subsubsection{General principles}

It is possible to measure the dynamic stiffness and damping of a mount enforcing a harmonic displacement expressed on equation 24 on the top of the mount and measuring the transmitted effort. Schematic of the test is presented Figure 2. We assume that the force transmitted is also harmonic with a delay as expressed in equation 25 .

$$
\begin{aligned}
& x_{0}(t)=X_{0} \cos (\omega t) \\
& f(t)=F \cos (\omega t+\delta)
\end{aligned}
$$

Using complex notation we obtain

$$
\begin{aligned}
& x_{0}^{*}(t)=X_{0} e^{i \omega t} \\
& f^{*}=F e^{i(\omega t+\delta)}
\end{aligned}
$$

The complex stiffness can be expressed as

$$
K^{*}=\frac{f^{*}}{x_{0}^{*}}=\frac{F}{X_{0}} e^{i \delta}=K^{\prime}+i K^{\prime \prime}
$$

This complex stiffness can be re-written as

$$
K^{*}=K^{\prime}(1+i \tan (\delta))
$$

With $\delta$, the phase of the complex stiffness defined by

$$
\delta=\operatorname{Arctan}\left(\frac{K^{\prime \prime}}{K^{\prime}}\right)
$$

The structural damping coefficient of the mount is then expressed as

$$
G=\tan (\delta)
$$

We can also define the dynamic stiffness

$$
K_{d y n}=\left|K^{*}\right|=\frac{F}{X_{0}}=\sqrt{K^{\prime 2}+K^{\prime \prime 2}}
$$


Figure 2 Dynamic stiffness and structural damping measurement

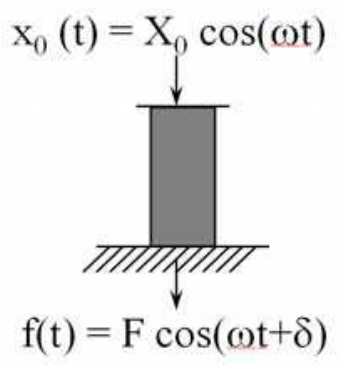

\subsubsection{Non-linear stiffness measurements}

Mounts are principally made of rubber. Those materials are non-linear with several parameters such as amplitude of excitation, temperature, static preload and frequency. We focused only on the non-linearity of the stiffness $K^{\prime}$ and of the structural damping coefficient $G$ due to the amplitude of excitation. There are several references dealing with the non-linearity with amplitude of elastomers and hyper-elastic materials (P. Saad (2003); A. Lion (1998)). Those phenomena are attributed to the friction between the long macromolecular chains constituting rubber. The more carbon black is present in the rubber, the more non-linear the mechanical properties. Measurements made on rubber samples (A. Lion (1998)) showed two principal effects of the amplitude of excitation on the mechanical properties of rubber:

- Stiffness decreases with amplitude of excitation (Payne Effect)

- Structural Damping coefficient first increases with amplitude and then decreases

In the following, we measured on a test bench the stiffness $K^{\prime}$ and structural damping $G$ along the principal direction for each mount that connects the engine to the car body. Before measuring, we applied to the engine mount and to the gearbox mount a static preload corresponding to the weight of the engine. Two parameters were considered : the frequency and the amplitude of excitation. For clarity, we fixed frequency at $25 \mathrm{~Hz}$ and we represented only the amplitudedependent normalized properties. Figure 3 and Figure 4 represent the evolution of the stiffness $K^{\prime}$ and of structural damping $G$, respectively. All curves were interpolated with a 6-degrees polynomial law. The obtained Root-Mean-Square coefficients are indicated. As observed on rubber samples, we notice that the dynamic stiffness increases as amplitude decreases while damping first increases with amplitude and decreases.

\subsection{Finite Element Model of the vehicle}

The Finite Element model of the vehicle includes the whole trimmed body, axles, wheels and the engine. This model counts millions of degrees-of-freedom and thus cannot be easily solved. In order to reduce matrix storage memory, we used a 
Figure 3 Stiffness K' interpolation for the four mounts
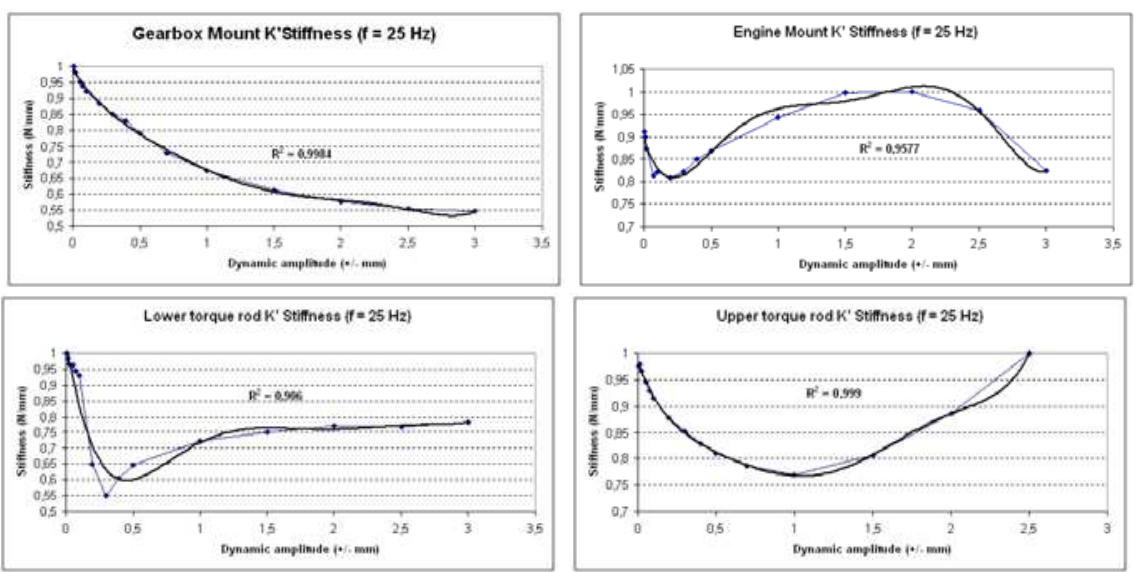

Figure 4 Structural damping coefficient interpolation for the four mounts
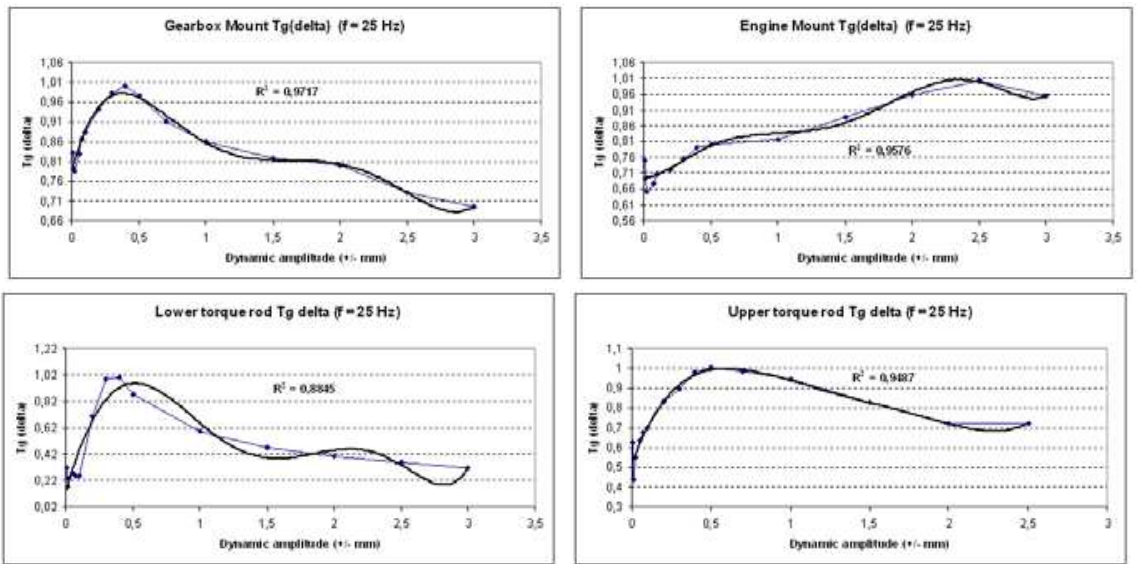

Craig and Bampton dynamic condensation method to reduce the vehicle only on few degrees-of-freedom containing the modal behaviour of the system.

\subsubsection{Craig and Bampton dynamic substructuring}

Craig and Bampton substructuring method (R.R. Craig and C.C. Bampton (1968);J.F. Imbert (1979)) is a well-known method for reducing the size of a problem. Starting from the stiffness matrix $\boldsymbol{K}$ of a mechanical system of size $N$, it is based on a partition of the degrees-of-freedom in two sets of size $B$ and $I$, so that $N=B+I$ :

- Boundary degrees-of-freedom (subscript B)

- Interior degrees-of-freedom (subscript I)

$$
\left\{\begin{array}{c}
\boldsymbol{F}_{B} \\
\boldsymbol{F}_{I}
\end{array}\right\}=\left[\begin{array}{cc}
\boldsymbol{K}_{B B} & \boldsymbol{K}_{B I} \\
\boldsymbol{K}_{I B} & \boldsymbol{K}_{I I}
\end{array}\right]\left\{\begin{array}{c}
\boldsymbol{X}_{B} \\
\boldsymbol{X}_{I}
\end{array}\right\}
$$


Considering fixed boundaries we assume that we can consider

$$
\left\{\boldsymbol{F}_{I}\right\}=\{\mathbf{0}\}
$$

Interior degrees-of-freedom can be expressed (after calculations) as

$$
\left\{\boldsymbol{X}_{I}\right\}=-\boldsymbol{K}_{I I}^{-1} \boldsymbol{K}_{I B}\left\{\boldsymbol{X}_{B}\right\}=[\boldsymbol{\Phi}]\left\{\boldsymbol{X}_{B}\right\}
$$

Applying the same partition to the lumped diagonal mass matrix $\boldsymbol{M}$ we obtain

$$
\boldsymbol{M}=\left[\begin{array}{cc}
\boldsymbol{M}_{B B} & 0 \\
0 & \boldsymbol{M}_{I I}
\end{array}\right]
$$

Substructure normal modes are defined as the normal modes of the structure with totally constrained boundaries. These are obtained from equations

$$
\begin{aligned}
& \left\{\boldsymbol{X}_{I}\right\}=\left\{\boldsymbol{\psi}_{I}\right\} e^{i \omega t} \\
& \omega^{2} \boldsymbol{M}_{I I}\left\{\boldsymbol{\psi}_{I}\right\}=\boldsymbol{K}_{I I}\left\{\boldsymbol{\psi}_{I}\right\}
\end{aligned}
$$

All the eigenvectors $\left\{\boldsymbol{\psi}_{I}\right\}$ are columns of the I-by-I matrix $\boldsymbol{\Psi}$ defined as the eigenvectors matrix of the fixed boundary system. Matrix $\boldsymbol{\Psi}$ is then truncated, keeping only the $r^{\text {th }}$ first eigenvectors defining matrix $\boldsymbol{\Psi}_{r}$ so that

$$
\boldsymbol{\Psi}=\left[\boldsymbol{\Psi}_{r} \boldsymbol{\Psi}_{I-r}\right]
$$

We also define the modal coordinates vector $\left\{\boldsymbol{\eta}_{r}\right\}$ so that

$$
\left\{\boldsymbol{X}_{I}\right\}=\left[\boldsymbol{\Psi}_{r}\right]\left\{\boldsymbol{\eta}_{r}\right\} e^{i \omega t}
$$

We can then express the coordinate transformation defined as

$$
\left\{\begin{array}{c}
\boldsymbol{X}_{B} \\
\boldsymbol{X}_{I}
\end{array}\right\}=\left[\begin{array}{cc}
\overline{\overline{\boldsymbol{I}}} & \overline{\overline{0}} \\
\boldsymbol{\Phi} & \boldsymbol{\Psi}_{\boldsymbol{r}}
\end{array}\right]\left\{\begin{array}{c}
\boldsymbol{X}_{B} \\
\boldsymbol{\eta}_{r}
\end{array}\right\} e^{i \omega t}=[\boldsymbol{\alpha}]\left\{\begin{array}{c}
\boldsymbol{X}_{B} \\
\boldsymbol{\eta}_{r}
\end{array}\right\}
$$

Where $\boldsymbol{\alpha}$ is a $B+I$-by- $B+r$ matrix

The stiffness matrix and the mass matrix after condensation are finally expressed as

$$
\begin{gathered}
\tilde{\boldsymbol{K}}=\boldsymbol{\alpha}^{T} \boldsymbol{K} \boldsymbol{\alpha} \\
\tilde{\boldsymbol{M}}=\boldsymbol{\alpha}^{T} \boldsymbol{M} \boldsymbol{\alpha}
\end{gathered}
$$

The matrices $\tilde{\boldsymbol{K}}$ and $\tilde{\boldsymbol{M}}$ are $B+r$-by- $B+r$ matrices. Such a method allows reducing considerably the number of unknowns representing the structure. In our case the initial model contains almost 4.5 millions degrees-of-freedom. By applying Craig and Bampton condensation method, the model is reduced to almost 500 degrees-of-freedom. 


\subsubsection{Application to the vehicle FE model}

First we apply Craig and Bampton condensation technique to the FE model of the vehicle defining

- $57 * 3$ boundary components

- 300 modal elements

Secondly, degrees-of-freedom are separated considering

- $9 * 3$ non-linear degrees-of-freedom : $8^{*} 3$ located on the 4 mounts (one on the engine side, the others on the body side) and $1^{*} 3$ at the center of gravity of the engine

- The other $48^{*} 3$ boundary components and the 300 modal degrees-of-freedom are defined as linear degrees-of-freedom

The whole FE model of the vehicle is presented Figure 5. The model after Craig and Bampton condensation method is presented Figure 6.

Figure 5 FE model of the vehicle before Craig and Bampton dynamic condensation

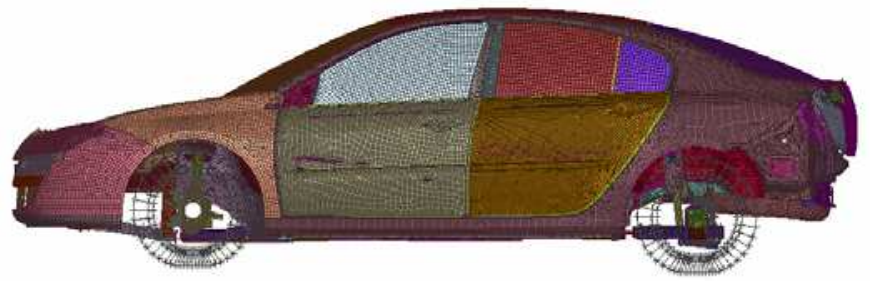

Figure 6 FE model of the vehicle after Craig and Bampton dynamic condensation

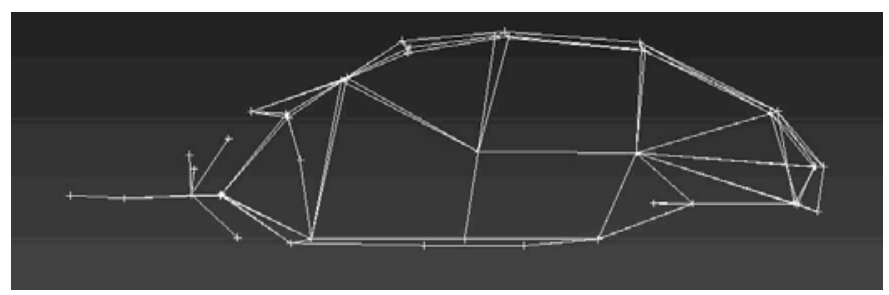

\subsection{Non-linear computation with an engine simple sine excitation}

We excited the system at the center of gravity of the engine with a simple sine force. In order to simplify the calculation, we only considered a one-stroke-perround excitation. The computation was done increasing the rotating speed $\Omega$ of the engine from 0 to $3000 \mathrm{RPM}$ which corresponds to 0 to $50 \mathrm{~Hz}$. This frequency range is the usual idle frequency range of excitation for thermal engines. Moreover, 
non-linear effects with amplitude are expected for low frequencies rather than high frequencies. In this frequency range the engine is assumed to be rigid, only represented by its mass and inertia. On the contrary, the car body presents lots of modes in this frequency range that have to be taken into account in the resolution. To simplify computations, we only used the interpolated non-linear functions measured for the stiffness $K^{\prime}$ at $25 \mathrm{~Hz}$. Structural damping coefficient $G$ is assumed to be constant with amplitude.

\subsubsection{Simple sine excitation}

The non-linear system is excited with a simple sine excitation at the center of gravity of the engine.

$$
F=F_{0} \sin (\Omega t)
$$

Where $\Omega$ is the rotating speed of the engine and $F_{0}$ the amplitude of the excitation fixed at $F_{0}=F_{\text {ref }}$. We first considered a small value for $F_{r e f}$. The excitation is along the vertical direction. As presented in Section 3.2.2 we performed a HBM computation with appropriate condensation on $9^{*} 3$ non-linear degrees-of-freedom. We considered the 5 first orders in the truncated Fourier series. The iterative solver was performed on those $9^{*} 3$ non-linear degrees-of-freedom. After convergence, the results were exported on the $48^{*} 3$ linear degrees-of-freedom. For each rotating speed of the engine and each degree-of-freedom $i$, we obtain the converged sine and cosine Fourier coefficients $\boldsymbol{A}_{k}^{i}$ and $\boldsymbol{B}_{k}^{i}$. For one value of the rotating speed $\Omega$, the total response of a degree-of-freedom $i$ can be estimated during a period $T=\frac{2 \pi}{\Omega}$.

$$
X^{i}(t)=\boldsymbol{B}_{0}^{i}+\sum_{k=1}^{m} \boldsymbol{A}_{k}^{i} \sin (k \Omega t)+\boldsymbol{B}_{k}^{i} \cos (k \Omega t) \quad t \in[0, T]
$$

Modulus of the total response is then defined as

$$
\left|X^{i}\right|=\max _{t \in[0, T]}\left(X^{i}(t)\right)
$$

We can also compute the response of order $k$ during a period which is given by

$$
\left|X_{k}^{i}\right|=\max _{t \in[0, T]}\left(\boldsymbol{A}_{k}^{i} \sin (k \Omega t)+\boldsymbol{B}_{k}^{i} \cos (k \Omega t)\right)
$$

After calculations we can show that

$$
\left|X_{k}^{i}\right|=\sqrt{\boldsymbol{A}_{k}^{i^{2}}+\boldsymbol{B}_{k}^{i^{2}}}
$$

Figure 7 presents the result of the calculation on the body side of the engine mount in the vertical direction. The sum of all orders and the amplitude of each order are plotted as functions of the rotating speed of the engine. We clearly see on this example that due to the non-linearities in the 4 mounts, harmonics appear in the solution. Amplitudes of the harmonics decrease and become less significant for high orders. We can notice that we find the first rigid body modes of the 
engine for low frequencies and car body modes for higher frequencies. For this low amplitude excitation, effects of the non linearity are small, since the non-linear (composite power) solution (black curve) is equal to the first order solution (blue curve) which means that the system can be considered as linear for this amplitude.

Another manner to represent the results is to plot the Fourier Coefficients of the truncated Fourier series for every rotating speed of the engine. We then construct a non-linear sonagram of the solution. For example, Figure 8 represents the sonagram obtained on the body side of the engine mount in the vertical direction. We can see rays appearing on the figure induced by the non-linearities in the mounts. For thermal engines rotating, such rays are basically visible on the response of the car body because engine excitations are multi-harmonic. In this case even a linear model reproduces the rays of the excitation. In our case, the rays are only due to the non-linearities present in the system excited by a single sine force. 
Figure 7 Total Response and order tracking for the engine mount (body side)

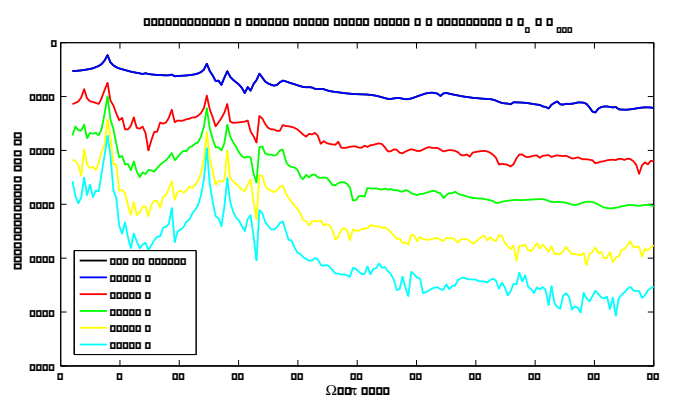

Figure 8 Non-linear sonagram for the engine mount (body side)

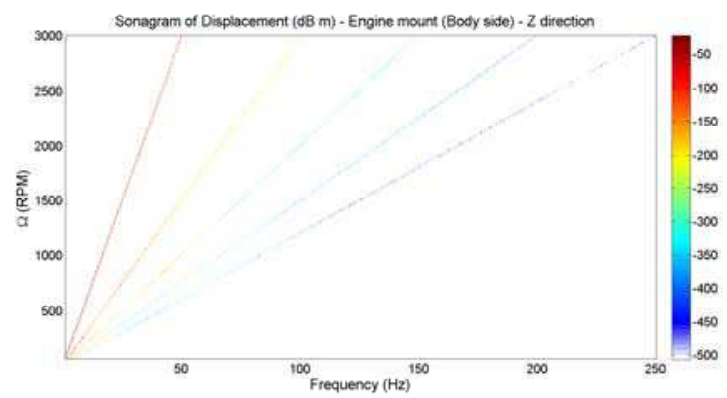

\subsubsection{Evolution with amplitude of excitation}

Considering same iterative stopping criteria, we compared the non-linear response of the car obtained for two various amplitudes of excitation with the corresponding linear solution obtained with a classical commercial software. Results are presented Figure 9 and Figure 10. Amplitudes chosen were $F_{0}=F_{\text {ref }}$ and $F_{0}=100 F_{\text {ref }}$.

First, we can note that for a low-amplitude excitation, we find the linear solution obtained with a commercial software, which validates the implementation of the HBM solver. Secondly, for higher amplitudes of excitation, we can note that the solution becomes more and more different from the linear solution.

Due to non-linear effects, we clearly see on Figure 10 that amplitude of the response is modified comparing with the corresponding linear solution. Globally, amplitude of the resonances at $5 \mathrm{~Hz}, 12 \mathrm{~Hz}$ and $15 \mathrm{~Hz}$ is decreasing with amplitude. Effects of non-linearities is also to shift the resonance frequencies lower than the linear solution $(5 \mathrm{~Hz})$ or higher $(12 \mathrm{~Hz}, 15 \mathrm{~Hz}, 18 \mathrm{~Hz})$. Small pics of resonance appear on the response $(2 \mathrm{~Hz})$ which correspond to the resonance of lower orders in the Fourier decomposition of the solution. 
Figure 9 Comparison of the linear and the non-linear solution for the engine mount (body side) $F_{0}=F_{\text {ref }}$

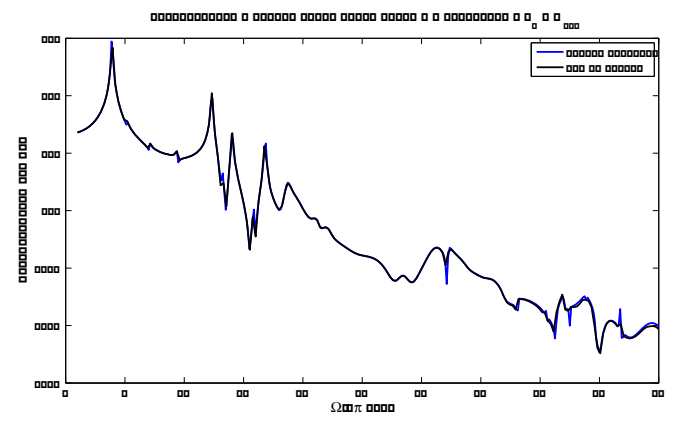

Figure 10 Comparison of the linear and the non-linear solution for the engine mount (body side) $F_{0}=100 F_{\text {ref }}$

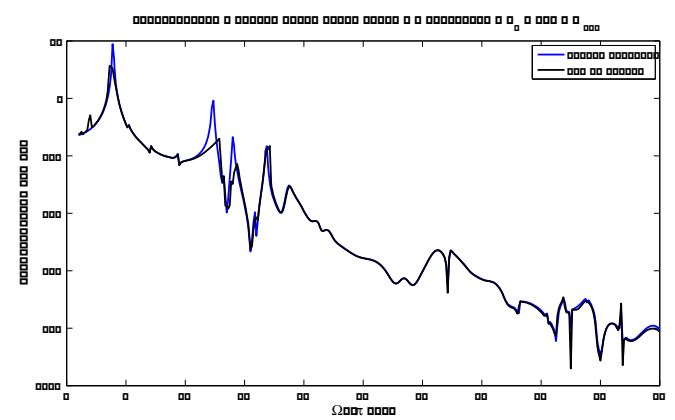

\subsubsection{Computational time considerations}

We can observe computer time needed for the previous calculations. For each amplitude, calculations were done for around 400 values of the rotating speed $\Omega$. Results are presented Table 1 . We can note that the higher the amplitude of excitation is, the longer computational time is. Computer time needed is not so huge comparing with classical industrial linear calculations.

We can compare computer time between results obtained with a HBM method without condensation process on the non-linear degrees-of-freedom and results obtained with a condensation process. Computer time was compared only calculating the response of the system for the first value of the engine rotating speed. Table 2 presents the results for the 2 previous amplitudes of excitation. We can note that the condensation process leads to the appropriate solution 300 times faster than classical HBM. For using HBM method on industrial models with many elements, condensation process is thus necessary. 
Table 1 Estimation of the CPU time for a HBM method with a condensation process

\begin{tabular}{lcc} 
& $F_{0}=F_{\text {ref }}$ & $F_{0}=100 F_{\text {ref }}$ \\
\hline HBM with condensation process & $7839 \mathrm{~s}$ & $12875 \mathrm{~s}$ \\
\hline
\end{tabular}

Table 2 Comparison of the HBM method without and with condensation process

\begin{tabular}{lcc}
\hline & $F_{0}=F_{\text {ref }}$ & $F_{0}=100 F_{\text {ref }}$ \\
\hline HBM without condensation process & $4969 \mathrm{~s}$ & $5038 \mathrm{~s}$ \\
\hline HBM with condensation process & $16 \mathrm{~s}$ & $19 \mathrm{~s}$ \\
\hline
\end{tabular}

\section{Conclusion}

In this paper we described the Harmonic Balance Method with a condensation process on the non-linear degrees-of-freedom. We applied the method to a whole vehicle FE model, containing non-linear engine mounts stiffness with amplitude of excitation. First, the FE model of the vehicle was reduced using a Craig and Bampton dynamic condensation method. Secondly the non-linear stiffness were measured on a test bench to evaluate non-linear polynomial laws. Finally, the non-linear system was excited by a simple sine excitation located at the center of gravity of the engine with various amplitude of excitation. Condensation process on the non-linear degrees-of-freedom allows huge reduction of computational time. We showed that for small amplitudes of excitation, non-linear effects were weak, while for higher amplitudes, non-linearities changed the response of the car, comparing to a classical linear solution. However, it may be noted that the comparison between numerical tests and experimental results may be very difficult to perform. For the problem of the dynamic response of a whole car, one of the most important factors is the connectors (for example welds) that are not usually modelled well. Comparison between numerical and experimental tests should be one of the future challenges for automotive industries.

\section{References}

Sinou, J.J. (2009) 'Non-linear dynamics and contacts of an unbalanced flexible rotor supported on ball-bearings', Mechanism and Machine Theory, Vol. 44,Elsevier (2009) pp.1713-1732.

Hahn, E.J. and Chen, P.Y. (1989) 'Harmonic Balance analysis of general squeeze film damped multi degree-of-freedom rotor bearing systems', Journal of tribology, Vol. 56 (1989), pp.149-154.

Jaumouillé, V., Sinou, J.J. and B. Petitjean (2010) 'An adaptive Harmonic Balance Method for predicting the nonlinear dynamic responses of mechanical systems Application to bolted structures', Journal of Sound and Vibration, Vol. 329, Issue. 19 (2010), pp.4048-4067.

J. Tezcan and J-K Hsiao (2008) 'Periodic solutions of the Duffing equation', Structural Engineering and Mechanics, Vol. 30, Issue. 5 (2008). 
Cameron, T.M., Griffin, J.H. (1989) 'An alternative frequency time domain method for calculating the steady state response of nonlinear dynamic systems', ASME Journal of Applied Mechanics, Vol. 56 (1989), pp.149-154.

Broyden, C.G. 'A class of methods for solving nonlinear simultaneous equations', Mathematics of computations, Vol. 116 (1994), pp.499-507.

Saad, P. 'Modélisation et identification du comportement non linéaire des cales en caoutchouc', Thèse de doctorat, Ecole Centrale de Lyon,(2003)

Lion, A. 'Thixotropic behaviour of rubber under dynamic loading histories: Experiments and theory', Journal of the Mechanics and Physics of Solids, Vol. 46, Issue. 5 (1998), pp.895-930.

Craig, R.R. and Bampton, C.C. 'Coupling of Substructures for Dynamic Analyses', AIAA Journal, Vol. 6, No. 7 (1968), pp.1313-1319.

Imbert, J.F. 'Analyse des structures par éléments finis, deuxième édition', Ecole Nationale Supérieure de l'Aeronautique et de l'Espace,(1979) 